TITLE:

\title{
A Hybrid System Approach to the Analysis and Design of Power Grid Dynamic Performance
}

\section{$\operatorname{AUTHOR}(\mathrm{S}):$}

Susuki, Yoshihiko; Koo, T. John; Ebina, Hiroaki; Yamazaki, Takuya; Ochi, Takashi; Uemura, Takuji; Hikihara, Takashi

\section{CITATION:}

Susuki, Yoshihiko ... [et al]. A Hybrid System Approach to the Analysis and Design of Power Grid Dynamic Performance. Proceedings of the IEEE 2012, 100(1):225-239

\section{ISSUE DATE:}

2012-01

URL:

http://hdl.handle.net/2433/152055

\section{RIGHT:}

(c) 2011 IEEE. Personal use of this material is permitted. Permission from IEEE must be obtained for all other uses, in any current or future media, including reprinting/republishing this material for advertising or promotional purposes,

creating new collective works, for resale or redistribution to servers or lists, or reuse of any copyrighted component of this work in other works.; This is not the published version. Please cite only the published version.; この論文は出版社版

でありません。引用の際には出版社版をご確認ご利用ください。 


\title{
A Hybrid System Approach to the Analysis and Design of Power Grid Dynamic Performance
}

\author{
Yoshihiko Susuki, Member, T. John Koo, Senior Member, Hiroaki Ebina, Non-Member, \\ Takuya Yamazaki, Non-Member, Takashi Ochi, Non-Member, Takuji Uemura, Non-Member, \\ Takashi Hikihara, Member
}

\begin{abstract}
We describe an approach to the analysis and design of power grid dynamic performance based on hybrid systems theory. Power grid is a large-scale Cyber-Physical System for transmission of electrical energy. The joint dynamics of physical processes and cyber elements in power grids are typical of a mixture of continuous and discrete behaviors, that is, hybrid dynamics. We address problems on stability that are basic concerns in the performance of current and future power grids with the hybrid dynamics. Measures for stability of power grids are interpreted as safety specifications in hybrid system models and are translated into restrictions on the systems' reachable sets of states. Algorithmic reachability analysis of hybrid systems enables analysis of safe initial states and hence quantitative estimation of stability regions. Also it contributes to synthesis of safe initial states as well as switching conditions in order to satisfy safety specifications in a power grid. We demonstrate the approach for two problems on transient stability of the single machine-infinite bus system and on fault release control of a multi-machine power grid.
\end{abstract}

Index Terms-cyber-physical system, power system, hybrid system, reachability, stability

\section{INTRODUCTION}

$\mathbf{P}$ OWER GRID is a large-scale Cyber-Physical System (CPS) [1]-[3] for transmission of electrical energy via a physical network from generation utilities to consumption loads overlaid with an information network for monitoring and control purposes. In the past decade, the large penetration of renewables such as solar and wind power generations, the aging power grid infrastructure, especially in the United States, and the emergence of global instabilities of power grids have advocated the use of modern Information and Communications Technology to change the current architecture and operation of power grids drastically. In particular, the Smart Grid [4] is introduced for a modernization of the electricity delivery system so it monitors, protects and automatically optimizes

Manuscript submitted April 5, 2011; revised July 3, 2011 and August 10, 2011; accepted August 20, 2011. This work was supported in part by Global COE Program on Photonics and Electronics Science and Engineering, MEXT, Japan, in part by NICT Project ICE-IT, and in part by National Natural Science Foundation of China, Grant No. 60773196.

Y. Susuki and T. Hikihara are with the Department of Electrical Engineering at Kyoto University, Katsura, Nishikyo, Kyoto, 615-8510 Japan (susuki@ieee.org, hikihara@kuee.kyoto-u.ac.jp).

T. J. Koo is with the Center for Embedded Software Systems, Shenzhen Institutes of Advanced Technology, Chinese Academy of Sciences, Shenzhen, 518055 China (john.koo@siat.ac.cn).

H. Ebina, T. Yamazaki, T. Ochi, and T. Uemura are with Kansai Electric Power Co., Inc., Japan (uemura.takuji@c2.kepco.co.jp). During parts of the work in this paper, H. Ebina was with the Department of Electrical Engineering at Kyoto University, Japan. the operation of its interconnected elements, and it will be characterized by a two-way flow of electricity and information to create an automated, widely distributed energy delivery network. The tight integration of the physical network for energy transmission and the information network is expected to enhance stability and reliability of the future power grid as in the Smart Grid.

The importance of joint dynamics of physical processes and cyber elements in power grids has increased drastically in recent years. They are defined as follows:

- Physical processes of a power grid are continuous-time behaviors of voltage, current, frequency, and power in dynamic components (generation plants, substations, automatic control systems, etc.) and in the whole physical transmission network of the grid.

- Cyber elements are those of computation, software, and networking for monitoring, analysis, and control of a power grid and aim to supervise the physical processes in the grid.

Recent examples of the cyber elements include the emergent system of wide-area monitoring, analysis, and control using Phasor Measurement Units (PMUs) that synchronize via global positioning system (see e.g. [5], [6]). In this case, the integration of cyber elements to the physical processes contributes the enhancement of grid stability and reliability. In [7], [8], the authors concern basic modeling of cyber-based physical energy systems and propose an interactive protocol between the controllers embedded within the system layers and the network operator to enable distributed sensing and actuation within this complex system.

Understanding the joint dynamics is the key enabler to realizing a stable and resilient power grid in the future. The joint continuous-discrete dynamics of physical processes and cyber elements in power grids can be modeled in the framework of hybrid systems [9]-[11]. Continuous-time dynamics describe the physical processes, and discrete event dynamics the behaviors of cyber components. In case that the physical processes of a power grid are only addressed, the dynamic performance is related to both linear analysis of it, characterized by small disturbance conditions, and the nonlinear situations that result from faults, major network changes, or loss of generation or load. On the other hand, the hybrid dynamics which we address in this paper can evolve in a complicated manner due to the integration of cyber elements to the physical processes. Currently, there is no systematic way 
to analyzing the hybrid dynamics, in particular, to defining and measuring stability of such cyber-physical power grids. The notion of stability is of basic importance in power grid dynamic performance [12]-[14] and is crucial for design of the future power grid. A new approach in the performance of power grids with the hybrid dynamics has been strongly required.

The purpose of this paper is to describe an approach to the analysis and design of power grid dynamic performance based on the theoretical foundations and computational methods developed for verification of hybrid systems. To verify a given system is to perform rigorous analysis for checking whether the system - or more accurately, the mathematical model describing the system-satisfies certain specifications [15], [16]. The notion of safety specifications is of basic importance for safety-critical applications such as flight management systems and transportation networks. Verifying a safety specification of a given system is to check whether the system can enter a specified set of unsafe states [15], [16]. Since many systems operate correctly only when being initialized correctly, a set of initial states is often specified. The so-called reachability analysis enables the check of safety specification [15], [16]: see Sec. III-B. In particular, we address the joint dynamics of physical processes and cyber elements via algorithmic approaches to the analysis and design issues. Basic algorithms developed here are directly implemented as cyber elements (computation) and utilized for analyzing and designing the physical processes. A connection between verification of engineered systems and hybrid operations of power grids is mentioned in [17].

The contributions of this paper are twofold. The first contribution is to show that the reachability analysis of hybrid systems enables quantitative estimation of stability in power grids. Since the notion of power grid stability is very broad [14], it is necessary to define appropriate measures for stability of interest. We interpret such measures for power grid stability as safety specifications in a hybrid model of power grid dynamic performance. Then, we translate the specifications into restrictions on reachable sets (see Sec. III-B) in the model's states. In this way, the reachable set computation and its software tools can be employed for the analysis of stability in a power grid, mathematically, the identification of safe initial states in the hybrid system model. We demonstrate our idea for two problems on transient stability. The so-called transient stability analysis is associated with the ability of a power grid to maintain synchronism after subjected to a large disturbance [12]-[14]. Loss of transient stability is recognized as one cause of the propagation of grid disturbances. Examples of the disturbance propagation include the September 2003 blackouts in North America and Italy [18]. Our approach is not specific to the transient stability problems and is applicable to various problems on stability, e.g., short-term voltage stability [19], [20]. The second contribution is to show that the reachability analysis enables the synthesis of safe initial states as well as switching conditions to satisfy safety specifications in a power grid. In [21] the authors developed an approach for estimating stability regions ${ }^{1}$ of continuous-time systems based on reachability computation. In [22] the authors computed stability regions of the continuous-time classical power system models using reachability computation. The approach is extended to hybrid systems for transient stability analysis with application in power systems (see Sec. IV). In this paper, we use the extended approach to synthesize safe initial states as well as switching conditions for given safety specifications that are based on proper operational regions. We demonstrate our idea for a problem on transient stability of a Single Machine-Infinite Bus (SMIB) system.

The organization of this paper is as follows. In Sec. II we provide review of related work. In Sec. III we present basic notions and computational aspects for hybrid systems that we will use throughout this paper. In Secs. IV and V we apply the methodology and tools for hybrid system reachability to two examples of transient stability problems in the SMIB system and a Double Machines-Infinite Bus (DMIB) system. In Sec. VI we conclude this paper with a summary and future directions. This paper is a revised and detailed version of the conference proceedings [23]-[27]. In Sec. IV-D we report a new result on the synthesis of safe initial states and switching conditions for a power grid. Also, in Sec. IV we describe algorithmic solutions for the analysis and design problems, which are different from the phenomenological approach in [24], [25] and are direct to the relevance and importance of CPS.

\section{RELATED WORK}

Many groups of researchers have been working at the intersection of research areas on power systems engineering and hybrid systems. In [28], [29], the authors use hybrid dynamical systems for modeling of power grid dynamics with considering transformer tap positions and relay internal states. A hybrid automaton model is also used in [30] for formulating inverse problems in power grids. In [31]-[33] the authors have attacked problems on voltage stability and control in power grids in terms of mixed logical dynamical systems and model predictive control. Also the approach based on model predictive control is reported in [34], [35]. In [36] the authors apply the hybrid input/output automaton to analysis of a power transmission system with relay control. In [37], [38], the authors study on-ship power system management using a hybrid system model. In [39] the authors use a hybrid dynamical system for modeling and analysis of the cascading failure leading to the 2003 blackout in Italy. In [40], [41] the authors use a hybrid automaton model for control of a microgrid with specifications of power balancing and stability. In [42] the authors discuss a hybrid system viewpoint in the VIKING project for resilient control of power networks. In [43] the authors study the issue of cyber attack to a power grid using the notion of hybrid system reachability.

\footnotetext{
${ }^{1}$ A stability region is defined as a basin of attraction of an attractor (normally, an asymptotically stable equilibrium) in a continuous-time dynamical system.
} 


\section{A Summarized Theory OF Hybrid Systems}

We summarize the theory of hybrid systems. The summary is based on [15], [16], [21] and contains basic notions and computation aspects: the model of hybrid automaton (Sec. III-A), safety verification and reachability analysis (Sec. III-B), and synthesis of safe initial states and switching conditions (Sec. III-C).

\section{A. Hybrid Automaton}

There are many mathematical models for hybrid systems that can represent the interaction between discrete event and continuous-time dynamics. We employ a hybrid automaton model for our purpose of research. One reason why we choose the hybrid automaton is that it is suitable for the purpose of multi-modal modeling, namely, continuous-time plants supervised by a hierarchical architecture of control systems. Such a control architecture is commonly used in real power grids: for example, emergency control and generation dispatching control.

The Hybrid Automaton (HA) is a formal model for a mixed discrete-continuous system [44]. Different groups of researchers have used different models for the HA. The HA model which we use in this paper is the following collection:

$$
\mathrm{H}=(\mathbb{Q}, \mathbb{X}, \Sigma, I, f, \text { Dom }, E, G),
$$

where

- $\mathbb{Q}$ is a finite set of discrete states. $\mathbb{X}$ is a set of continuous states. We refer to the pair $(q, x) \in \mathbb{Q} \times \mathbb{X}$ as the state of $\mathrm{H}$;

- $\Sigma$ is a set of discrete input symbols;

- $I \subseteq \mathbb{Q} \times \mathbb{X}$ is a set of initial states;

- $f: \mathbb{Q} \times \mathbb{X} \rightarrow \mathrm{TX}$ assigns to every discrete state a Lipschitz continuous vector field on $\mathbb{X}$, where $T \mathbb{X}$ is the tangent bundle of $\mathbb{X}$;

- Dom : $\mathbb{Q} \rightarrow P(\mathbb{X}) \times \Sigma$ is called a domain and defines combinations of states and inputs for which continuous evolution is allowed. $P(\mathbb{X})$ is the power set (set of all subsets) of $\mathbb{X}$;

- $E \subseteq \mathbb{Q} \times \mathbb{Q}$ is a collection of edges (discrete transitions);

- $G: E \times \Sigma \rightarrow P(\mathbb{X})$ is called a guard condition for discrete transition and defines transition relations of discrete states.

Note that in the current model (1) we do not consider continuous inputs and disturbances, discrete disturbance symbols, and reset functions that appear in other models of HA. This is just for ease of presentation and clarity. Often it is required to consider these for solving control problems on power grid dynamics: see [40], [41]. Also note that trajectories in the current model (1) of HA evolve continuously as well as in discrete jumps. Existence and uniqueness of trajectories in $\mathrm{H}$ are a challenging subject by itself (see [45] and references therein). In this paper, to pay our attention on hybrid systems technology, we do not investigate the mathematical subject of hybrid system trajectories. A mathematical notion of trajectories in $\mathrm{H}$ is summarized in [15].

\section{B. Safety Verification and Reachability Analysis}

Safety verification of $\mathrm{H}$ is to perform rigorous analysis to check whether trajectories of $\mathrm{H}$ can enter a specified target set of (unsafe) states. The problem of safety verification poses as a property of the reachable set of states [15], [16]. Define a target set for $\mathrm{H}$ by $T \subseteq \mathbb{Q} \times \mathbb{X}$. There are two notions of reachable sets in literature: forward and backward reachable sets. A forward reachable set is a collection of all possible trajectories of $\mathrm{H}$ departing from $I$. A backward reachable set is a collection of all possible trajectories of $\mathrm{H}$ arriving at $T$. Safety verification can be done by analyzing these reachable sets. If the forward reachable set intersects $T$, then the system whose design and behavior are modeled by $\mathrm{H}$ is unsafe. If the backward reachable set intersects $I$, then the system is also unsafe. Thus the computation of reachable sets does work for verifying system safety.

Computation of reachable sets is a widely active research field in computer science and control engineering: see [16] and references therein. In [46] the authors introduce a HamiltonJacobi-Issac equation as a mathematical formulation of (backward) reachable sets in HA models. In the following, we review an algorithmic reachability analysis for continuoustime systems based on the Level Set Method (LSM) [47], [48]. The analysis method is proposed in [15], [49] and can be extended for hybrid systems: see [15], [49] and Secs. IV-C and IV-D. Consider a continuous-time model described by $f(x)$ in $\mathrm{H}$ :

$$
\frac{\mathrm{d} x}{\mathrm{~d} t}=f(x),
$$

where $x \in \mathbb{X}$ and $t \geq 0$. We use $\left.T\right|_{\mathbb{X}}$ to represent a target set for (2) defined as

$$
\left.T\right|_{\mathbb{X}}=\{x \in \mathbb{X} ; J(x, 0) \leq 0\},
$$

where $J: \mathbb{X} \times \mathbb{R} \rightarrow \mathbb{R}$ is termed the value function. The LSM treats time evolutions of the boundary of $\left.T\right|_{\mathbb{X}}$, denoted by $\partial\left(\left.T\right|_{\mathbb{X}}\right)$, as the zero level set of the value function $J(x, t)$ at each moment $t$, that is, $\{x \in \mathbb{X} ; J(x, t)=0\}$. The time evolution of $\partial\left(\left.T\right|_{\mathbb{X}}\right)$ is represented by the following Level Set Equation (LSE):

$$
\frac{\partial J}{\partial t}+(\nabla J)^{\top} f(x)=0,
$$

where $\nabla J$ is the derivative of $J$ with respect to $x$, and $\top$ is the transpose operation of vectors. Hence, the forward reachable set of (2) departing from $\left.T\right|_{\mathbb{X}}$ with time duration $\Delta t$ is represented as

$$
\begin{aligned}
\text { Post }_{\Delta t}\left(\left.T\right|_{\mathbb{X}}, f(x)\right):= & \{x \in \mathbb{X} ; \exists \tau \in[0, \Delta t], \\
& J(x, \tau) \leq 0\} .
\end{aligned}
$$

Since the model (2) is time-invariant, the backward reachable set of (2) arriving at $\left.T\right|_{\mathbb{X}}$ with time duration $\Delta t$ is also represented as

$$
\begin{aligned}
\operatorname{Pre}_{\Delta t}\left(\left.T\right|_{\mathbb{X}}, f(x)\right):= & \{x \in \mathbb{X} ; \exists \tau \in[0, \Delta t], \\
& \left.J^{\prime}(x, \tau) \leq 0\right\},
\end{aligned}
$$

where $J^{\prime}(x, t)$ is the solution of (4) obtained by replacing $f(x)$ with $-f(x)$. Thus the forward and backward reachable sets can be computed by solving the corresponding LSEs. 
In [21] the authors demonstrate that the LSM can be used for computation of reachable sets as well as stability regions of (2). The computation procedure is summarized in Algorithm 1. In the algorithm, starting from the initial set $S_{0}$, the backward reachable set is computed iteratively for an integer multiple of $\Delta t$. The algorithm terminates due to the following two reasons. Firstly, the algorithm terminates at a fixed point solution, i.e. $S_{k+1} \subseteq S_{k}$ for some $k$, which indicates there is no new state that can be reached. Also, the termination can also happen due to the finite representation of reachable sets on a Cartesian grid. Secondly, if the number of iterations exceeds the predefined limit $\mathcal{T} / \Delta t$, the algorithm will terminate due to the second condition. For the initial set $S_{0}=\left.T\right|_{\mathbb{X}}$, the proposed algorithm attempts to synthesize a subset of $\mathbb{X}$ such that if a state departs from the subset, it can eventually reach $\left.T\right|_{\mathbb{X}}$ (at most) in the bounded time $\mathcal{T}$. The executable code in each computation is automatically generated by the ReachLab platform [50] and deployed in a quad-core machine for implementation.

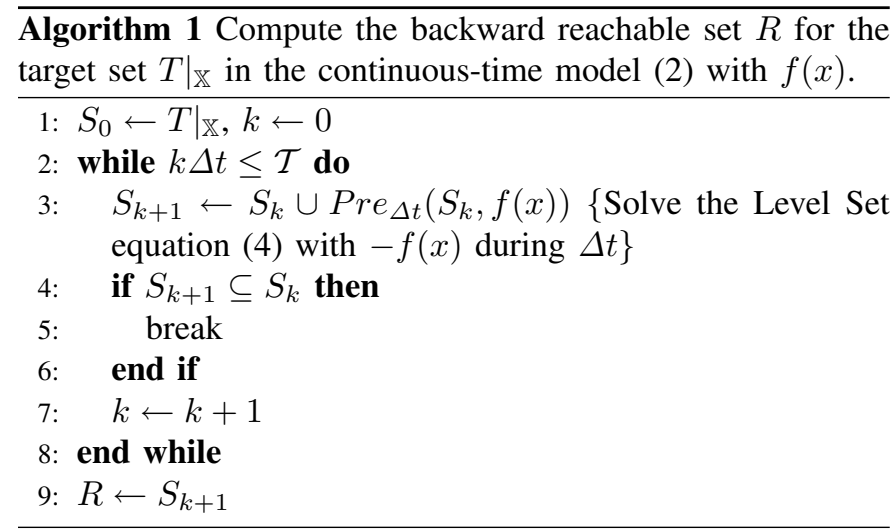

\section{Reachability Analysis and System Design}

Lastly, we introduce a reachability-based approach to the design of hybrid systems. This approach enables the design of hybrid systems that satisfy reachability specifications. For given initial set $I$ and final set $F \subseteq \mathbb{Q} \times \mathbb{X}$, we are now interested in checking whether there exist some $(q(0), x(0)) \in I$ and $\tau>0$ such that $(q(\tau), x(\tau)) \in F$, where $(q(t), x(t))$ denotes a trajectory of $\mathrm{H}$ starting from $(q(0), x(0))$ at time $0 .{ }^{2}$ This is one type of reachability specifications for $\mathrm{H}$. First, by using the forward reachability algorithm, we analyze the socalled mode switching problem [51], that is, we find a finite sequence of discrete states as well as switching conditions by which a trajectory of $\mathrm{H}$ departing from $I$ reaches $F$. Second, if $F$ is reachable from $I$, then we apply the backward reachability algorithm departing from the intersection of the forward reachable set and the final set $F$ to go backward. Here, the forward reachable set for $I$ in $\mathrm{H}$ is denoted by $\operatorname{Post}(I, \mathrm{H})$ in the same manner as in (5), and the backward reachable set is denoted by $\operatorname{Pre}(F \cap \operatorname{Post}(I, \mathrm{H}), \mathrm{H})$ as in (6). Lastly, by refining the guard condition $G$ in $\mathrm{H}$ and the

\footnotetext{
${ }^{2}$ Here we have implicitly assumed that the trajectory exists in a (finite) domain of state and time which we are interested of.
}

initial set $I$ with the backward reachable set, we synthesize the safe guard condition $G_{\text {safe }}$ between discrete states as well as the safe initial set $I_{\text {safe }}$. The set $I_{\text {safe }}$ corresponds to $I \cap \operatorname{Pre}(F \cap \operatorname{Post}(I, \mathrm{H}), \mathrm{H})$. With the procedure, we are able to refine the HA model to satisfy the reachability specification, given as $\mathrm{H}_{\text {safe }}$ :

$$
\mathrm{H}_{\mathrm{safe}}=\left(\mathbb{Q}, \mathbb{X}, \Sigma, I_{\mathrm{safe}}, f, D o m, E, G_{\mathrm{safe}}\right) .
$$

\section{Transient Stability Analysis of the Single MACHINE-INFINITE BUS SYSTEM WITH RE-Closing OPERATION}

In this section, we apply the algorithmic reachability analysis of HA models to analysis of transient stability of the Single Machine-Infinite Bus (SMIB) system. The SMIB system is shown in Fig. 1 and contains one synchronous machine, the infinite bus ${ }^{3}$, and two parallel transmission lines connecting the machine with the infinite bus. In Sec. IV-A we introduce the re-closing operation for the SMIB system and formulate two problems on transient stability. In Sec. IV-B we develop a HA model that represents the dynamical interaction between continuous-time behaviors of the synchronous machine and the re-closing control. In Secs. IV-C and IV-D we perform the reachability analysis for the model and solve the two problems.

\section{A. Re-Closing Operation and Problem Statements}

An electromechanical oscillation of the synchronous machine is a core cause of loss of transient stability. The oscillation is triggered by a severe disturbance or fault in the SMIB system (such as lightning attack and timber contact) and is affected by control of relay devices equipped with the transmission lines. Fig. 1 shows two operating conditions or modes of the SMIB system that emerge due to the fault and control sequence:

- 1-line operation (a) is the mode after clearing a faulted line by relay device.

- 2-lines operation (b) is the mode after re-closing the faulted line. The mode corresponds to that before the occurrence of fault.

The purpose of the current transient stability analysis is to analyze the effect of re-closing operation, that is, a switching

(a)

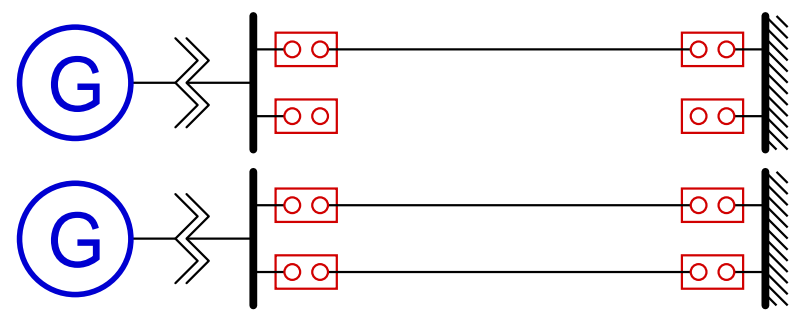

Fig. 1. Single Machine-Infinite Bus (SMIB) system and its two modes (operating conditions) that we analyze in Sec.IV: (a) 1-line operation mode and (b) 2-lines operation mode. The switching between the modes results in hybrid dynamics of the SMIB system.

\footnotetext{
${ }^{3} \mathrm{An}$ infinite bus is a source of voltage constant in phase, magnitude, and frequency, and is not affected by the amount of current withdrawn from it [12]
} 
from 1-line operation mode to 2-lines operation mode, on the electromechanical oscillations of the synchronous machine. Examples of the effect are large excursion of rotor speed of the machine and its loss of transient stability with respect to the infinite bus ${ }^{4}$. Since the standard transient stability analysis does investigate dynamics in one of the modes, it corresponds to the identification of stability region in a continuous-time model. On the other hand, the current analysis in this section (and the next section, too) involves multiple modes and a switching logic between them. The two problems which we solve in this paper are stated as follows:

1) For given re-closing operation (mode switching) and initial states, specify a subset of the initial states in which the synchronous machine does not show any loss of transient stability.

2) Determine a timing of the re-closing operation by which the synchronism machine can avoid the loss of transient stability.

In the current SMIB system, since the only two modes are addressed, the first analysis problem is not hard to solve and is appropriate for demonstration of our approach. The second control problem has been not fully discussed from an analytical point of view, because it is inevitable to tackle hybrid dynamics arising in power grids. Note that in [25] we consider the case of three modes when adding the new mode, called the fault-on operation.

\section{B. Hybrid Automaton Model}

For the stated problems, continuous-time dynamics in $\mathrm{H}$ model the electromechanical oscillation of the synchronous machine. Discrete event dynamics model the transition between the two modes due to the re-closing operation. The HA model (1) makes it possible to combine the continuoustime dynamics with the discrete event dynamics. For the continuous-time dynamics, we use $\delta$ to represent the rotor position of the machine with respect to synchronous reference axis (the infinite bus) and $\omega$ to represent the non-dimensional rotor speed deviation relative to system angular frequency $(2 \pi \times 50 \mathrm{~Hz}$ or $2 \pi \times 60 \mathrm{~Hz})$. The electromechanical oscillation of the machine is represented by the nonlinear swing equations [12]:

$$
\frac{\mathrm{d} \delta}{\mathrm{d} t}=\omega, \quad \frac{\mathrm{d} \omega}{\mathrm{d} t}=p_{\mathrm{m}}-b \sin \delta-k \omega .
$$

The parameters $p_{\mathrm{m}}, b$, and $k$ are constant in time and are in per unit system. The constant $p_{\mathrm{m}}$ is the mechanical input power to the machine, $b$ the critical transmission power between the machine and the infinite bus, and $k$ the damping coefficient in the SMIB system. The constant $b$ is proportional to the number of parallel AC transmission lines. Next, we model the two modes of operation in Fig. 1, using the two discrete states $q_{1}$ and $q_{2}$. The state $q_{1}$ is assigned to the 1-line operation mode, and $q_{2}$ to the 2-lines operation mode. The re-closing operation is modeled as the discrete control symbol $\sigma$. Hence,

\footnotetext{
${ }^{4}$ This is called stepping-out phenomenon or loss of synchronism.
}

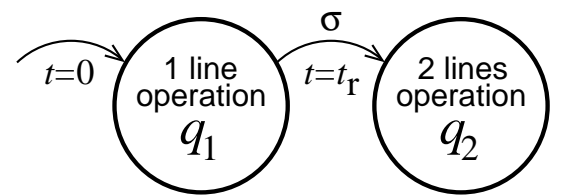

Fig. 2. Hybrid automaton $\mathrm{H}$ including one discrete control input which represents the re-closing operation

the interaction of the electromechanical oscillation with the re-closing operation is represented by $\mathrm{H}$ :

$$
\begin{aligned}
& \mathbb{Q} \times \mathbb{X} \quad=\left\{q_{1}, q_{2}\right\} \times\left(\mathbb{T}^{1} \times \mathbb{R}^{2}\right), \\
& (\delta, \omega, z) \in \mathbb{X} \\
& \Sigma \quad=\{\sigma\}, \\
& I \quad \text { (fixed later), } \\
& f\left(q,(\delta, \omega, z)^{\top}\right)=\left(\begin{array}{c}
\omega \\
p_{\mathrm{m}}-\alpha b \sin \delta-k \omega \\
1
\end{array}\right) \\
& \text { at } \begin{cases}\alpha=0.5 & \text { if } q=q_{1}, \\
\alpha=1 & \text { if } q=q_{2},\end{cases} \\
& \operatorname{Dom}(q) \quad=\mathbb{X} \times \Sigma, \\
& E \quad=\left\{\left(q_{1}, q_{2}\right)\right\}, \\
& G\left(\left(q_{1}, q_{2}\right), \sigma\right)=\left\{(\delta, \omega, z) \in \mathbb{X} ; z=t_{\mathrm{r}}\right\}
\end{aligned}
$$

where $t_{\mathrm{r}}$ stands for the onset time of re-closing operation. The continuous state space $\mathbb{X}$ in $H$ contains a timer $z \in \mathbb{R}$ in order to force the discrete transition. The continuous vector field $f$ is given by the nonlinear swing equations (8) and is parameterized by $\alpha$ that depends on the discrete states. The schematic diagram of $\mathrm{H}$ including the discrete control input is shown in Fig. 2.

In order to solve the current problems, we define the unsafe target set $T$, its boundary $\partial T$, and the set $I$ of initial states as

$$
\left.\begin{array}{rl}
T & =\left\{q_{1}, q_{2}, q_{3}\right\} \times\left. T\right|_{\mathbb{X}}, \\
\left.T\right|_{\mathbb{X}} & =\left\{(\delta, \omega, z) \in \mathbb{X} ;|\omega|>\omega_{\mathrm{c}}\right\}, \\
\partial T & =\left\{q_{1}, q_{2}, q_{3}\right\} \times\left\{(\delta, \omega, z) \in \mathbb{X} ;|\omega|=\omega_{\mathrm{c}}\right\}, \\
I & =\left\{q_{1}\right\} \times\left\{(\delta, \omega, z) \in \mathbb{X} ;|\omega|<\omega_{\mathrm{c}}, z=0\right\},
\end{array}\right\}
$$

where $\omega_{\mathrm{c}}$ is a threshold value of the rotor speed deviation $\omega$ of synchronous machine. The states in $T$ represent unacceptable operations of the machine such as large rotor speed deviation and stepping-out phenomenon of it. In the nonlinear swing equations (8), the stepping-out phenomenon of the machine is represented as a stable limit cycle of the second kind [52], which goes arounds the cylindrical phase space and is located at $\omega \approx p_{\mathrm{m}} / k$. In order to avoid the large rotor speed and stepping-out, it is natural to fix $\omega_{\mathrm{c}}$ at the values that do not exceed the location. In this way, the unsafe target set $T$ provides an appropriate measure of loss of transient stability in the SMIB system .

\section{Reachability Computation and Analysis of Transient Sta- bility}

In the first analysis problem, we are interested in checking whether the synchronous machine can survive without any 
loss of transient stability with the re-closing operation. Since the associated dynamics are hybrid, it is required to extend Algorithm 1 to the HA model for the SMIB system with the re-closing operation. Here, because the discrete transition is triggered by time only, the basic structure of Algorithm 1 remains. The continuous-time dynamics need to be changed once the discrete transition happens. This is possible by inserting if-then statements in Algorithm 1. The modified procedure of reachable set computation is summarized in Algorithm 2.

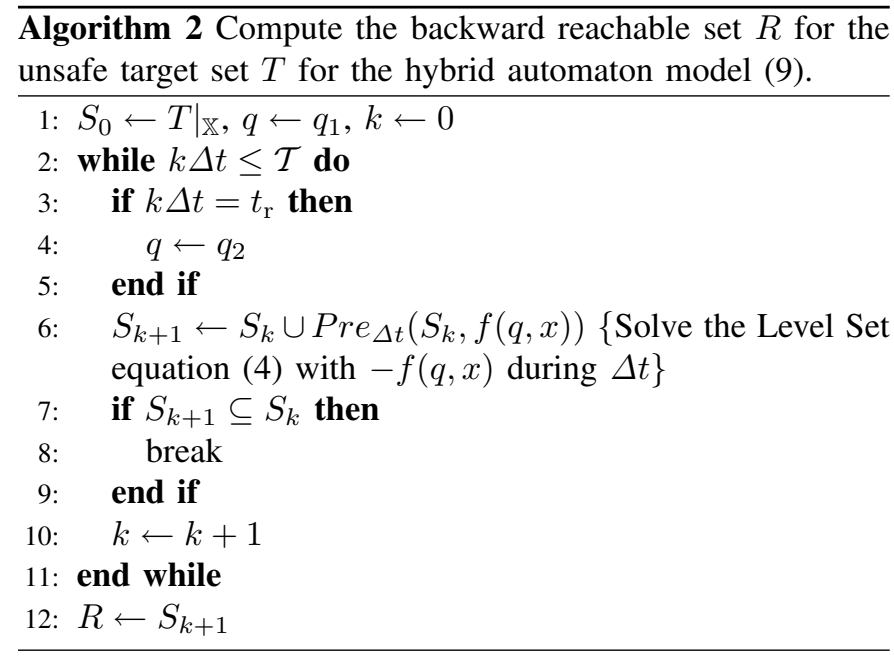

Figure 3 shows a numerical result of backward reachable sets based on Algorithm 2. We use the following values of the constant parameters in (8):

$$
p_{\mathrm{m}}=0.2, \quad b=0.7, \quad k=0.05 .
$$

We limit the analysis set $\mathbb{X}$ to $[-\pi, \pi] \times[-2,2]$, and fix the grid size $\Delta x$ as $0.05 \times 0.05$ and the time step $\Delta t$ as 0.01 . Under the numerical setting, the time consumption for obtaining each of the figures was about 3 hours. In each figure, the noncolored (white) region corresponds to the safe initial states that is indirectly estimated by Algorithm 1. Within this region it is guaranteed that the synchronous machine does not show any loss of transient stability. The red closed loop, denoted by 'stability limit,' stands for the sufficient condition based on the closest u.e.p. method [13] for the 1-line operation mode and indeed becomes a sufficient condition in the case of HA model. Compared with the conventional method, the reachabilitybased method provides less conservative condition for the HA model. $R_{\text {before }}$ is the subset of $\mathbb{X}$ from which any state reaches to $\partial T$ before the re-closing operation, and $R_{\text {after }}$ is the one from which any state reaches to $\partial T$ after the re-closing. The numerical result coincides with the previous one in [24] based on the direct numerical integration of the HA model. Thus the LSM is effective for the estimation of transient stability of the SMIB system with a switching control. Fig. 3 also shows how the reachable set changes as the re-closing time $t_{\mathrm{r}}$ increases. The parameter $t_{\mathrm{r}}$ determines the impact of the re-closing operation to the transient stability. As $t_{\mathrm{r}}$ increases, the non-colored region approaches the sufficient condition of stability region in the continuous-time model, which is based

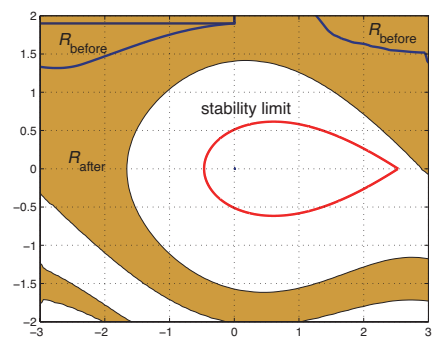

(a) $t_{\mathrm{r}}=0.1 \mathrm{~s} / t_{\mathrm{b}}$

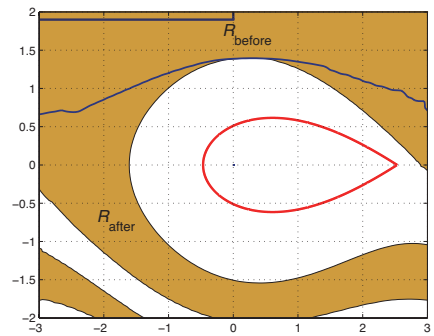

(c) $t_{\mathrm{r}}=0.5 \mathrm{~s} / t_{\mathrm{b}}$

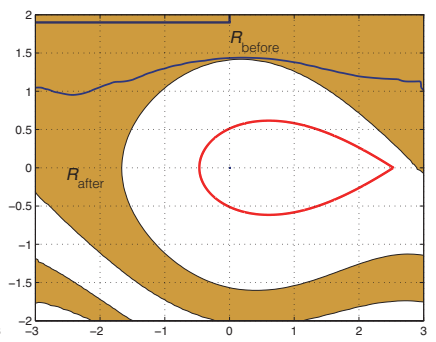

(b) $t_{\mathrm{r}}=0.3 \mathrm{~s} / t_{\mathrm{b}}$

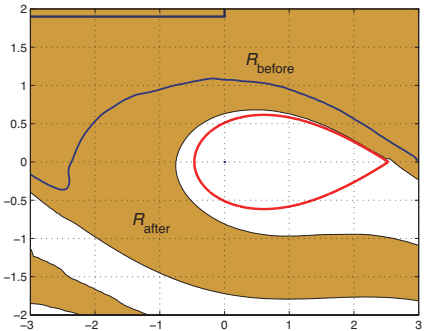

(d) $t_{\mathrm{r}}=0.8 \mathrm{~s} / t_{\mathrm{b}}$
Fig. 3. Backward reachable sets in the hybrid automaton model representing the re-closing operation. The horizontal axis denotes the rotor position $\delta$, and the vertical axis the rotor speed deviation $\omega$. The parameter $t_{\mathrm{r}}$ is the re-closing time and controls when the re-closing operation occurs in the 1-line operation mode.

on the closest u.e.p. method. This is exactly true because the limit of infinite $t_{\mathrm{r}}\left(t_{\mathrm{r}} \rightarrow \infty\right)$ yields the continuous case without the re-closing operation.

\section{Reachability Computation and Synthesis of Safe Initial States}

To solve the second control problem in Sec. IV-A, we use the approach introduced in Sec. III-C to synthesize a set of safe initial states of the SMIB system as well as switching conditions. Since in the current model the discrete transition $\sigma$ is triggered by time only, the mode switching problem is nothing particular. Therefore we concentrate on the synthesis of safe initial states and switching conditions.

Figure 4 shows another result of backward reachable sets for the HA model (9). The procedure for the computation is as follows. We limit the analysis set $\mathbb{X}$ to $[-\pi, \pi] \times[-\pi, \pi]$. Also we fix the initial set $I$ as $\mathbb{X}$ at the 1-line operation mode $q_{1}$ and the final set $F$ as the ball $B_{r}\left(x_{0}\right)$ with center $x_{0}=(0.289752,0)$ and radius $r=0.2$. The center $x_{0}$ corresponds the steady operating state of the machine at $q_{2}$, that is, the asymptotically stable equilibrium of the continuoustime model at $q_{2}$. We also fix the grid size $\Delta x$ as $0.025 \times 0.025$ and the time step $\Delta t$ as 0.5 . When the SMIB system is in the 2-lines operation mode $q_{2}$, the dynamics of the system are governed by (8) at $q_{2}$. The reachable set evolves in backward time until it reaches a fixed-point solution of the corresponding LSE or terminates due to a predefined limit. The final time is denoted by $t_{1}=180$, and the final set $R_{1}$ is marked in yellow. Then we switch the SMIB system to the 1-line operation mode $q_{1}$. Along the dynamics of the system described by (8) at $q_{1}$, the backward reachable set $R_{2}$ to $R_{1}$ is obtained and marked in red. This set corresponds to a fixed-point solution of the 


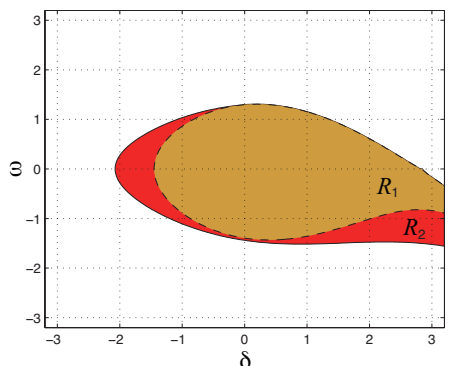

Fig. 4. Backward reachable sets and synthesis of safe initial states. $R_{1}$ denotes the reachable set to a small neighborhood of a stable equilibrium of the continuous-time model (8) at $q_{2}$, and $R_{2}$ the reachable set to $R_{1}$ along the dynamics described by (8) at $q_{1}$. The union of $R_{1}$ and $R_{2}$ results in a subset of safe initial states in the SMIB system.

LSE for $q_{1}$. The final time $t_{2}$ for obtaining $R_{2}$ is 60 , and the total time of computation is $t_{1}+t_{2}=240$. The procedure is summarized in Algorithm 3.

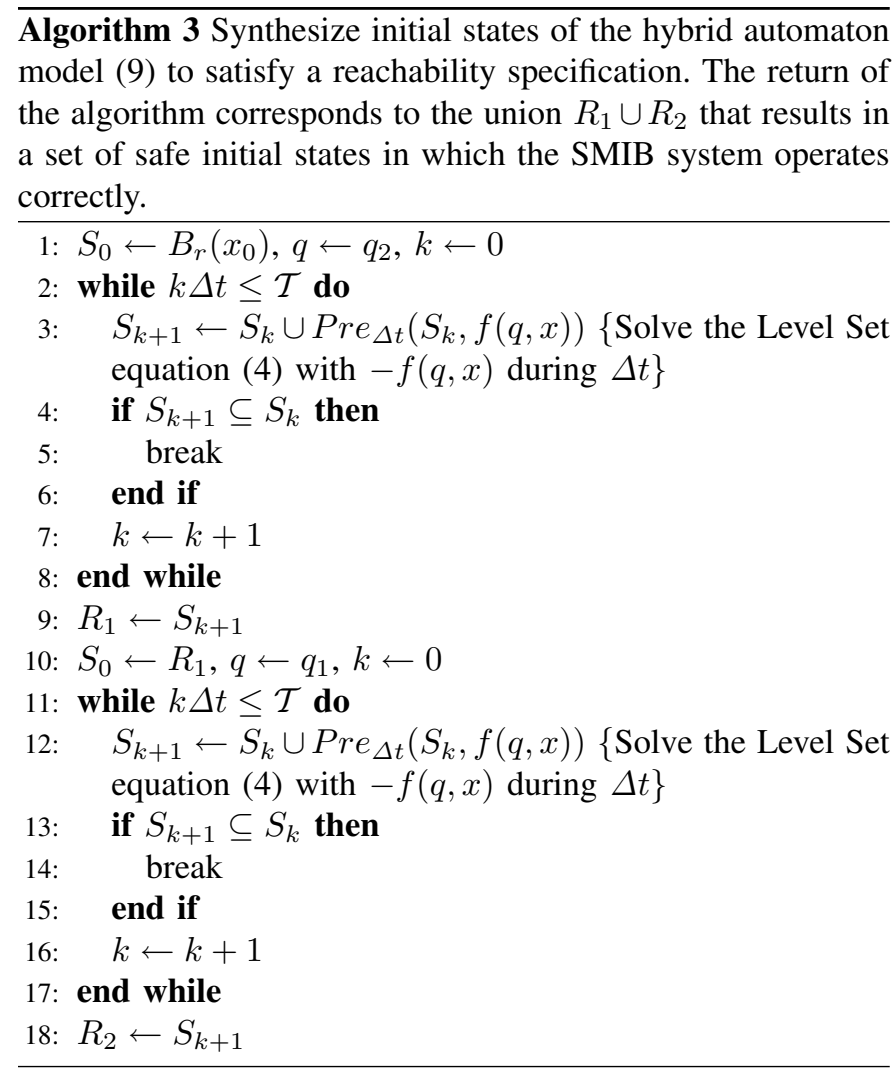

Now we investigate in details the result of backward reachable sets shown in Fig. 4. Since $R_{2}$ does not reach the unsafe target set $T$, it corresponds to a set of safe initial states which can ensure the reach to the 2-lines' safe set. The union of the two reachable sets $R_{1}$ and $R_{2}$ becomes a set of safe initial states in the SMIB system, that is, $I_{\text {safe }}$ in the context of Sec. III-C. Thus the backward reachability algorithm enables the synthesis of safe initial states. In addition to the safe state synthesis, we see a safe switching condition to guarantee that the synchronous machine does not show any loss of transient stability. Since $R_{2}$ does not touch $T$, the discrete transition $\sigma$, namely the switching from 1-line operation mode to 2-lines operation mode, can occur wherever the 2-lines' safe set is reached. The switching guarantees the system's safety, that is, the synchronous machine does not show any loss of transient stability.

\section{E. Concluding Remarks}

We solved the problems on transient stability of the SMIB system with the re-closing operation. The main concern is how we analyze the effect of the re-closing operation to the dynamics of the synchronous machine. An HA model is used for representing the dynamical effect of the re-closing operation. The reachability analysis of the HA model specifies a set of initial states in which the synchronous machine does not show any loss of transient stability with respect to the infinite bus. Also the analysis makes it possible to determine the timing of the re-closing by which the machine also does not show the loss of transient stability.

\section{Verification of Fault Release Control FOR A DOUble MACHINES-INFINITE Bus SySTEM}

As the second example of the application, we consider the problem on verification of fault release control in a multimachine power grid. In Sec. V-A we introduce a Double Machines-Infinite Bus (DMIB) system and the basic concept of fault release control. In Sec. V-B we review a practical data of the fault release control and state a problem on the verification of fault release control. In Secs. V-C and V-D we analyze the problem by using a HA model and its forward reachability analysis. The details of the contents presented here are given in [26].

\section{A. Protection Control Mechanisms}

The DMIB system is shown in Fig. 5 and consists of two synchronous generators, the infinite bus, an AC transmission network, loads, relays, and circuit breakers. Arrows in the figure represent constant power loads. The two synchronous generators, denoted by G1 and G2, are connected via the transmission network. The DMIB system has the three protection systems, each of which consists of relay and circuit breaker. Two of the protection systems are equipped with G1 and G2 and denoted by the green boxes in Fig. 5. The other protection system is denoted by the red boxes in line 5 . Now we introduce two control mechanisms for these protection systems as follows.

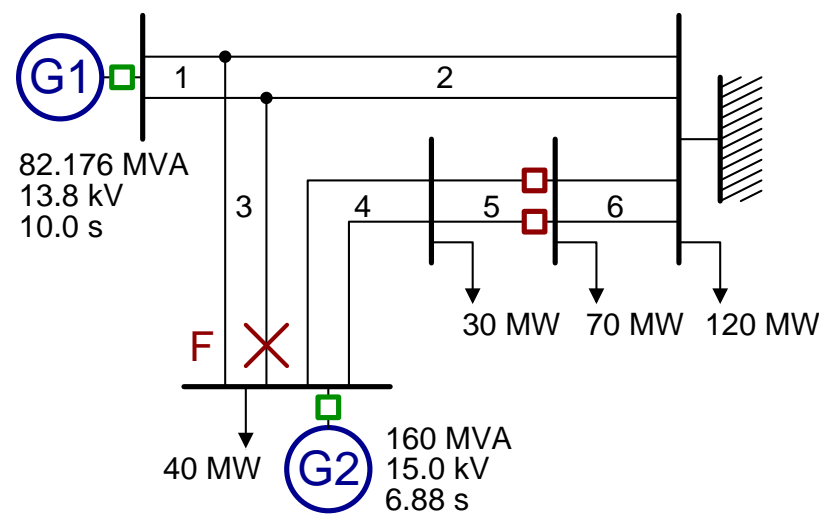

Fig. 5. Double Machines-Infinite Bus (DMIB) system that we analyze in Sec. V. The system is based on a practical configuration in the Japanese power grid. 
1) Generation Trip Control: Each of the protection systems with G1 and G2 consists of an impedance-type relay and a circuit breaker. The protection system for G1 (or G2) tries to remove G1 (or G2) from the network if it exhibits a signature of phenomena in loss of transient stability. The signature here is monitored with a trajectory of complex-valued net impedance of the network measured from each generator bus. If the impedance passes the imaginary axis from right domain of complex plane to left, then the circuit breaker trips, that is, removes a generator from the network. The objective of the control mechanism is to remove a generator exhibiting the loss of transient stability quickly and to keep the whole DMIB system stable. The protection system is regarded as a decentralized controller with single continuous-valued input and single discrete-valued output.

2) Fault Release Control: The protection system equipped with line 5 executes the fault release control. The objective of the control is to mitigate an electrical stress of the whole DMIB system caused by disturbances and to keep the whole system stable. The circuit breaker can trip, that is, disconnect the line in a prescribed period (normally a few cycles) after either generator is removed by the above protection system. In this way, the onset of breaker trip for fault release control depends on that of generation trip. The protection system is regarded as a centralized controller with single discrete-valued output.

\section{B. Practical Data and Problem Statement}

In this sub-section we present a practical data on the fault release control in the DMIB system. The data is obtained with the RTDS (Real Time Digital Simulator) produced by RTDS Technologies Inc. RTDS is a fully digital electromagnetic transient power system simulator (testbed). In the simulator, the dynamics of a synchronous generator are modeled by the twoaxis Park equations with damper windings. Detailed effects of automatic voltage regulator (AVR) and power system stabilizer (PSS) can be considered. In this way, we are referring to a simulation result obtained with RTDS as a practical data. However, for simplicity of the current analysis, we do not assume that AVR and PSS operate in the current simulation. We use the following fault condition. Each generator operates at a steady operating state at $t<t_{\mathrm{f}}=0 \mathrm{~s}$. Then a three-phase fault happens at point $\mathrm{F}$ (see Fig. 5) near G2 bus at $t=t_{\mathrm{f}}=0 \mathrm{~s}$, and the faulted line trips at $t=t_{\mathrm{cl}}=8 /(60 \mathrm{~Hz}) \sim 0.133 \mathrm{~s}$. The fault duration corresponds to 8 cycles of $60-\mathrm{Hz}$ sinusoidal wave. Also we assume that the fault release control is executed in $0.3 \mathrm{~s}$ after either $\mathrm{G} 1$ or $\mathrm{G} 2$ is removed by its protection system. The setting of parameters in the DMIB system, which includes data on synchronous generators and $\mathrm{AC}$ transmission lines, is presented in [26]. The parameters are used for the RTDS analysis.

Figure 6 shows a data on rotor speed deviations $\omega_{1}$ of $\mathrm{G} 1$ and $\omega_{2}$ of $\mathrm{G} 2$ relative to the nominal angular frequency $(2 \pi \times$ $60 \mathrm{~Hz}$ ). The figure (a) is for $\mathrm{G} 1$ and (b) for G2. The figures are reproduced from [26]. The figure (a) contains the two transient behaviors denoted by Case 1 and Case2. Case1 represents the transient behavior of $\mathrm{G} 1$ without fault release control, and

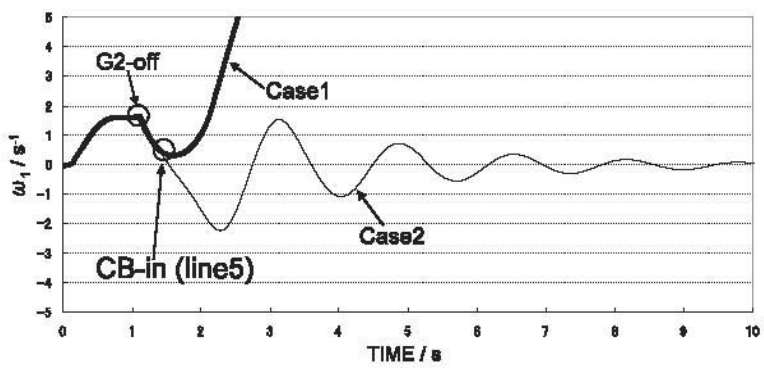

(a) Rotor speed deviation $\omega_{1}$ of $\mathrm{G} 1$

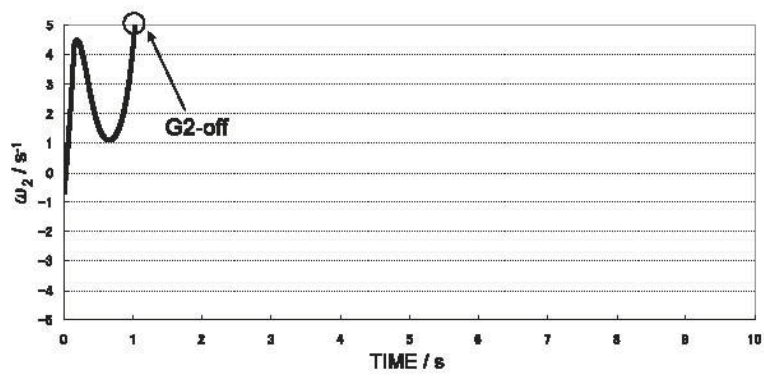

(b) Rotor speed deviation $\omega_{2}$ of $\mathrm{G} 2$

Fig. 6. Practical data on fault release control for the Double Machines-Infinite Bus (DMIB) system. The figures are reproduced from [26].

Case2 the transient behavior with it. The onset of the control is denoted by CB-in (line5). G2-off represents the onset of G2 trip caused by the protection system. In Fig. 6(b) G2 exhibits a divergence motion of $\omega_{2}$, that is, loss of transient stability that does not depend on the fault release control. On the other hand, in Fig. 6(a) G1 does not exhibit any divergence motion of $\omega_{1}$ under the fault release control and finally settles down a steady operating condition. These figures suggest that the fault release control can avoid the loss of transient stability of G1.

The problem which we analyze in this section is to prove the correctness of the fault release control in a framework of hybrid systems reachability. Apparently, the dynamics associated with the problem are hybrid due to the dynamical interaction between electromechanical oscillations of the two machines and the discrete protection controls. In the following, with the HA model and reachability computation, we will consider the problem on hybrid dynamics and control of a multi-machine power grid.

\section{Hybrid Automaton Model}

Now we derive a HA model that represents the dynamical interaction between electromechanical oscillations of the two machines and the discrete protection controls. First, we model the electromechanical oscillations of G1 and G2. The oscillations are represented by the continuous-time vector field $f$ in (1). In the same manner as in Sec. IV-B, we use $\delta_{i}$ to represent the rotor position of $\mathrm{G} i$ with respect to synchronous reference axis (the infinite bus) and also use $\omega_{i}$ to represent the non-dimensional rotor speed deviation of $\mathrm{G} i$ relative to system angular frequency $\omega_{\mathrm{s}}=2 \pi \times(60 \mathrm{~Hz})$. The electromechanical oscillations of $\mathrm{G} i(i=1,2)$ are represented by the nonlinear 
swing equations:

$$
\left.\begin{array}{c}
\frac{\mathrm{d} \delta_{i}}{\mathrm{~d} t}=\omega_{i}, \\
a \frac{2 H_{i}}{\omega_{\mathrm{s}}} \frac{\mathrm{d} \omega_{i}}{\mathrm{~d} t}=P_{\mathrm{m} i}-D \omega_{i}-G_{i i} V_{i}^{2}-\sum_{j \in\{1,2, \infty\} \backslash\{i\}} \\
\left\{G_{i j} V_{i} V_{j} \cos \left(\delta_{i}-\delta_{j}\right)+B_{i j} V_{i} V_{j} \sin \left(\delta_{i}-\delta_{j}\right)\right\},
\end{array}\right\}
$$

The variable $\delta_{\infty}$ is the rotor position of infinite bus and is assumed to be zero. The parameters $a, H_{i}, P_{\mathrm{m} i}, D, G_{i i}$, $G_{i j}, B_{i j}$, and $V_{i}$ are constant in time and are in per unit system except for $H_{i}$ and $D$ in seconds. The constant $a$ stands for the ration of generator rated capacity of $\mathrm{G} 1$ to that of $\mathrm{G} 2$ and appears in the swing equations for G1 only. The constant $H_{i}$ is the per unit inertia constant of $\mathrm{G} i$, and $D$ is the damping coefficient of generators. The constant $G_{i i}$ is the internal admittance, and $G_{i j}+\mathrm{j} B_{i j}$ the transfer admittance. The constants $V_{1}$ and $V_{2}$ stand for the bus voltages of $\mathrm{G} 1$ and $\mathrm{G} 2$, and $V_{\infty}$ the voltage of infinite bus.

Second, we model the protection systems of generation trip and fault release control. The modeling which we now perform is similar to that in [39]. The parameters $G_{i i}, G_{i j}$, and $B_{i j}$ in (12) discontinuously change when the fault, generation trip, and line trip happen. For the HA model (1), we need to describe all possible network topologies explicitly. However, when the current goal is to follow the data in Sec. V-B, it is enough to introduce the following small set $\mathbb{Q}$ consisting of four discrete states $\left\{q_{1}, q_{2}, q_{3}, q_{4}\right\}$. The state $q_{1}$ is assigned to the fault-on operating condition, namely, mode, $q_{2}$ to the mode after removing the faulted line, $q_{3}$ to the mode after removing G2 by generation trip, and $q_{4}$ to the mode after fault release control. In fact, there are no other modes that appear in the following numerical analysis. Here we re-write the continuoustime model (12) to explicitly clarify its dependency of the modes. In the right-hand side of (12), the third terms represent the output power of $\mathrm{G} i$ and depend on the modes. Thus the output power, denoted by $P_{\mathrm{e} i}^{(q)}$ with index $q \in \mathbb{Q}$, is given as

$$
\begin{aligned}
P_{\mathrm{e} i}^{(q)}= & G_{i i}^{(q)} V_{i}^{2}+\sum_{j \in\{1,2, \infty\} \backslash\{i\}}\left\{G_{i j}^{(q)} V_{i} V_{j} \cos \left(\delta_{i}-\delta_{j}\right)\right. \\
& \left.+B_{i j}^{(q)} V_{i} V_{j} \sin \left(\delta_{i}-\delta_{j}\right)\right\},
\end{aligned}
$$

where $i=1,2$. The parameter $G_{i i}^{(q)}$ represents the internal admittance of $\mathrm{G} i$ for index $q$, and similarly for $G_{i j}^{(q)}, B_{i j}^{(q)}$. In this modeling, we represent the generation trip and fault release control themselves as discrete control inputs in $\Sigma$. For the data presented in Sec. V-B, the protection control which removes the faulted line is modeled as the control input $\sigma^{1}$, and it drives the discrete transition from $q_{1}$ to $q_{2}$. The protection control which removes G2 is modeled as $\sigma^{2}$, and it drives the discrete transition from $q_{2}$ to $q_{3}$. The fault release control is modeled as $\sigma^{3}$, and it drives the discrete transition from $q_{3}$ and $q_{4}$.

Lastly, we integrate the continuous-time dynamics in (12) with the discrete transitions above and derive a HA model used for analysis of the verification problem. The HA model

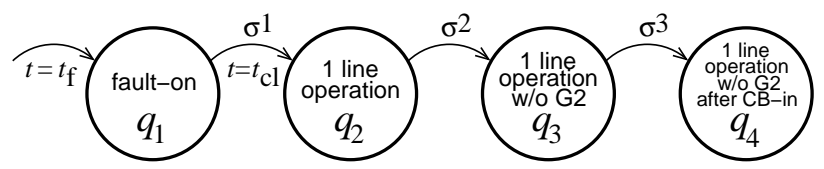

Fig. 7. Hybrid automaton model $\mathrm{H}$ including three discrete control inputs that represent the line clearing, generation trip control, and fault release control in a Double Machines-Infinite Bus (DMIB) system

is the following.

$$
\begin{array}{ll}
\mathbb{Q} & =\left\{q_{1}, q_{2}, q_{3}, q_{4}\right\} \\
\mathbb{X} & =\mathbb{T}^{2} \times \mathbb{R}^{3} \ni\left(\delta_{1}, \delta_{2}, \omega_{1}, \omega_{2}, z\right)=: x, \\
\Sigma & =\left\{\sigma^{1}, \sigma^{2}, \sigma^{3}\right\} \\
f(q, x), & q \in \mathbb{Q} \\
E & =\left\{\left(q_{1}, q_{2}\right),\left(q_{2}, q_{3}\right),\left(q_{3}, q_{4}\right)\right\} \\
\operatorname{Dom}(q) & =\mathbb{X} \times \Sigma, \\
G\left(\left(q_{i}, q_{i+1}\right), \sigma^{i}\right), i=1,2,3
\end{array}
$$

A schematic diagram of $\mathrm{H}$ is Fig. 7. The new variable $z / \mathrm{s} \in \mathbb{R}$ with continuous-time dynamics $\dot{z}=1$ is added to $\mathbb{X}$ in order to drive the discrete control inputs $\sigma^{1}$ and $\sigma^{3}$. The vector field $f(q, x)$ is given by

$$
f(q, x)=\left(\begin{array}{c}
\omega_{1} \\
\omega_{2} \\
\frac{\omega_{\mathrm{s}}}{2 H_{1} a}\left\{P_{\mathrm{m} 1}-D \omega_{1}-P_{\mathrm{e} 1}^{(q)}(x)\right\} \\
\frac{\omega_{\mathrm{s}}}{2 H_{2}}\left\{P_{\mathrm{m} 2}-D \omega_{2}-P_{\mathrm{e} 2}^{(q)}(x)\right\} \\
1
\end{array}\right) .
$$

The guard condition $G$ is based on the control mechanisms of the protection systems and is defined as

$$
\begin{aligned}
G\left(\left(q_{1}, q_{2}\right), \sigma^{1}\right)= & \left\{x \in \mathbb{X} ; z=t_{\mathrm{cl}}\right\}, \\
G\left(\left(q_{2}, q_{3}\right), \sigma^{2}\right)= & \left\{x \in \mathbb{X} ; \operatorname{Re}\left[Z_{2}(x)\right]=0\right. \text { and } \\
& \left.\mathrm{d}\left(\operatorname{Re}\left[Z_{2}(x)\right]\right) / \mathrm{d} t<0\right\}, \\
G\left(\left(q_{3}, q_{4}\right), \sigma^{3}\right)= & \left\{x \in \mathbb{X} ; z=t_{2}+\Delta t\right\},
\end{aligned}
$$

where $Z_{2}(x)$ is the complex-valued net impedance of the $\mathrm{AC}$ transmission network measured from G2 bus. The real part of $Z_{2}(x)$ is crucial to the current modeling and is given by

$$
\begin{aligned}
\operatorname{Re}\left[Z_{2}\right]= & \frac{V_{2}}{\left|I_{2}^{\left(q_{2}\right)}\right|^{2}}\left\{\operatorname { c o s } \delta _ { 2 } \sum _ { j \in \{ 1 , 2 , \infty \} } V _ { j } \left(G_{2 j}^{\left(q_{2}\right)} \cos \delta_{j}\right.\right. \\
& \left.-B_{2 j}^{\left(q_{2}\right)} \sin \delta_{j}\right)+\sin \delta_{2} \sum_{j \in\{1,2, \infty\}} V_{j}\left(G_{2 j}^{\left(q_{2}\right)} \sin \delta_{j}\right. \\
& \left.\left.+B_{2 j}^{\left(q_{2}\right)} \cos \delta_{j}\right)\right\}
\end{aligned}
$$

The complex-valued output current $I_{2}^{\left(q_{2}\right)}$ of $\mathrm{G} 2$ is a function of rotor positions $\left(\delta_{1}, \delta_{2}\right)$ and discrete states $q_{i}$. For $G\left(\left(q_{3}, q_{4}\right), \sigma^{3}\right)$ in (16), the time $t_{2}$ is the onset of discrete control $\sigma^{3}$, and $\Delta t$ is the prescribed delay time for the fault release control. 


\section{Reachability Computation and Verification of Fault Re- lease Control}

In this sub-section we use the notion of forward reachable set of $\mathrm{H}$ in order to analyze the correctness of fault release control. For this, we need to fix a unsafe target set $T$ and a set of initial states $I$ that are appropriate for the current problem. The choice of $T$ depends on the aim of control mechanism whose performance we want to verify. Since the aim of fault release control is to avoid the loss of transient stability of G1, the unsafe target set $T$ can be defined as

$$
T=\left\{(q, x) \in \mathbb{Q} \times \mathbb{X} ; \omega_{1} \geq \omega_{\text {cr }}\right\},
$$

where $\omega_{\text {cr }}(>0 \mathrm{rad} / \mathrm{s})$ is the critical value of rotor speed deviation $\omega_{1}$ under which G1 can operate safely. The reason why we use $\omega_{\text {cr }}$ is that if G2 is removed from the network, the loss of transient stability in G1 is well represented by solutions converging to the stable periodic solution of the second kind, located at $\omega_{1}(t) \sim P_{\mathrm{m} 1} / D(>0)$, in (12). The choice of $I$ depends on how initial states we consider for the DMIB system. The data in Sec. V-B is obtained under the assumption at $t=t_{\mathrm{f}}$ that the DMIB system operates at a steady state. With these in our mind, we use the following setting of $I$ :

$$
\begin{aligned}
I= & \left\{(q, x) \in \mathbb{Q} \times \mathbb{X} ; q=q_{1}, z=t_{\mathrm{f}},-\pi \leq \delta_{1}<\pi,\right. \\
& -5 \mathrm{rad} / \mathrm{s} \leq \omega_{1} \leq 5 \mathrm{rad} / \mathrm{s}, \delta_{2}=\delta_{2}^{*}, \\
& \left.\omega_{2}=0 \mathrm{rad} / \mathrm{s}\right\},
\end{aligned}
$$

where $\delta_{2}^{*}$ is the value at the steady operating state, in this case, an asymptotically-stable equilibrium of the continuoustime model (12) before the fault. The set $I$ contains the stable equilibrium. Here we attempt to analyze the correctness of fault release control under perturbations to the initial conditions. This is why $I$ does has non-zero measure.

Here, different from the algorithmic approach in Sec.IV, we perform the reachability analysis using direct numerical integration of (12). The set $I$ are finely partitioned by a grid. The trajectory starting from each element of the grid is computed by numerical integration and is checked whether it intersects with $T$ or not. The parameters $G_{i i}$ and $G_{i j}+\mathrm{j} B_{i j}$ change at the onset of discrete transitions and are presented in [26]. The other parameters are also presented in [26]. Note that this numerical approach is not rigorous, but it will explain the practical data in Sec. V-B.

Figure 8 shows the numerical result on forward reachable sets for the HA model. In the two figures, each bounded, two-dimensional plane corresponds to the initial set $I$. The colored regions are related to non-empty intersections of the forward reachable sets departing from $I$ and $T$. Each of the regions is a finite set of elements of the grid from which trajectories of $\mathrm{H}$ intersect with $T$ in time $5 \mathrm{~s}$. The non-colored (white) regions represent the subset of $I$ from which trajectory does not intersect with $T$ for $5 \mathrm{~s}$. Fig. 8(a) is the case without fault release control, namely, without the discrete control $\sigma^{3}$. The symbol IP represents a steady operating condition and corresponds to the initial state used for the RTDS analysis in Sec. V-B. The initial state IP in Fig. 8(a) exists inside the colored region. This implies that as time passes, G1 will exhibit the loss of transient stability without fault release

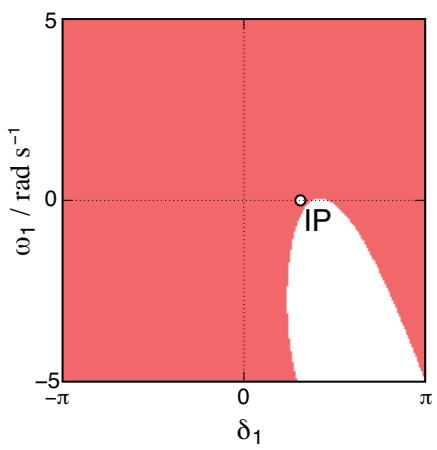

(a) Case1

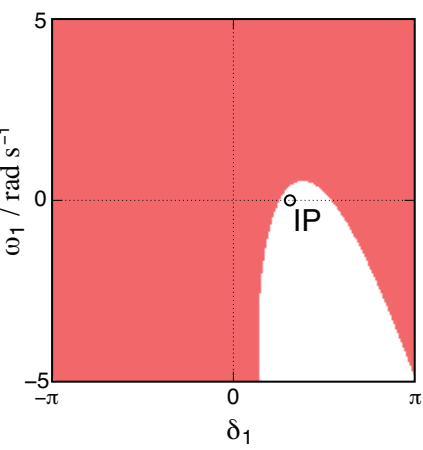

(b) Case2
Fig. 8. Numerical results of reachability analysis of the hybrid automaton model (14). The colored regions are the subsets of $I$ in which the forward reachable sets departing from $I$ and the unsafe target set $T$ intersect. Case 1 is for the result without fault release control, and Case 2 for the result with fault release control.

control. This is consistent with the practical data in Fig. 6(a). On the other hand, Fig. 8(b) is the case with the fault release control $\sigma^{3}$. The colored region represents an approximation the subset of $I$ from which trajectory intersects with $T$ at $q_{i}(i=1,2,3)$ in $5 \mathrm{~s}$. The symbol IP also represents the initial state used for the RTDS analysis and exists outside the colored region, i.e., the forward reachable set. This implies that by the fault release control, G1 does not show the loss of transient stability in $5 \mathrm{~s}$. This is also consistent with the practical data in Fig. 6(a). Hence the reachability analysis of the HA model indicates that the fault release control is correct for prevention of loss of transient stability in G1.

\section{E. Concluding Remarks}

We used the reachability analysis of hybrid systems for analyzing the verification problem of fault release control in the DMIB system. The problem is intended to verify a practical data of fault release control that is obtained with the RTDS analysis. The fault release control implies a state-dependent mechanism and needs to be accurately modeled as a hybrid controller. An HA model is used for modeling the hybrid controller and representing its dynamical effect on the whole system. The forward reachability analysis of the HA model suggests the correctness of the fault release control that can avoid the loss of transient stability of all of the machines in the system.

\section{Summary AND Future Directions}

We presented an approach to the analysis and design of power grid dynamic performance. The approach is based on the theoretical foundations and computational methods developed for the verification of hybrid systems and is emergent by an integration of ideas from control engineering, computer science, and power systems engineering. We have demonstrated the integration for the two problems on transient stability in the SMIB and DMIB systems. These show that the application of reachability analysis of hybrid system models enables the quantitative estimation of transient stability and the 
synthesis of safe initial states as well as switching conditions to satisfy safety specifications.

In this paper the reachable set computation was applied to the analysis and design of power grid dynamic performance. Since we intend to contain a right balance between tutorial state-of-the-art exposition and research, prototype examples of transient stability problems in small-scale power grids are only addressed. It is important to see the application of our approach to large-scale problems where stability depends on system-wide parameters. For a large-scale power grid, computational costs for the reachable set computation naturally increase. There are several directions to mitigate these costs: to project the reachable set of a high-dimensional system into a collection of lower dimensional subspaces [53], and to design an architecture of distributed reachability computation that overlays the physical architecture of a power grid. Scalability of the reachable set computation is of basic importance in the latter direction of this research.

\section{ACKNOWLEDGMENTS}

We are grateful to Mr. Kosaku Yokota and Mr. Osamu Yatsubo (Kansai Electric Power Co., Inc.) for valuable discussions on power systems engineering, to Mr. Rongqing $\mathrm{Li}$ (Chinese Academy of Sciences) for his assistance on reachable set computation, to Mr. Masataka Minami for his careful reading of the manuscript, and to anonymous reviewers for their valuable suggestions.

\section{REFERENCES}

[1] Cyber-Physical Systems Executive Summary, Prepared by the CPS Steering Group, March 6, 2008.

[2] N. Adam, Final Report of Workshop on Future Directions in CyberPhysical Systems Security, Department of Homeland Security, January 2010.

[3] R. Poovendran, "Cyber-physical systems: Close encounters between two parallel worlds," Proc. IEEE, vol. 98, no. 8, pp. 1363-1366, August 2010.

[4] Electric Power Research Institute, Report to NIST on the Smart Grid Interoperability Standards Roadmap (Contract No. SB1341-09-CN-0031), June 17, 2009.

[5] A. G. Phadke, "Synchronized phasor measurement in power systems," IEEE Comput. Appl. Power, vol. 6, no. 2, pp. 10-15, April 1993.

[6] J. De La Ree, V. Centeno, J. S. Thorp, and A. G. Phadke, "Synchronized phasor measurement applications in power systems," IEEE Trans. Smart Grid, vol. 1, no. 1, pp. 20-27, June 2010.

[7] M. D. Ilić, L. Xie, U. A. Khan, and J. M. F. Moura, "Modeling future cyber-physical energy systems," in Proc. IEEE PES General Meeting, Pittsburgh, USA, July 2008.

[8] — "Modeling future cyber-physical energy systems for distributed sensing and control," IEEE Trans. Syst., Man, Cybern. A, vol. 40, no. 4, pp. 825-838, July 2010.

[9] R. Alur, C. Courcoubetis, N. Halbwachs, T. A. Henzinger, P. H. Ho, X. Nicollin, A. Olivero, J. Sifakis, and S. Yovine, "The algorithmic analysis of hybrid systems," Theor. Comput. Sci., vol. 138, no. 1, pp. 3-34, February 1995.

[10] E. Clarke, A. Fehnker, Z. Han, B. Krogh, J. Ouaknine, and O. Stursberg, "Abstraction and counterexample-guided refinement in model checking of hybrid systems," Int. J. Found. Comput. Sci., vol. 14, no. 4, pp. 583604, August 2003.

[11] "Special Issue on Hybrid Systems: Theory and applications," Proc. IEEE, vol. 88, no. 7, pp. 879-1123, July 2000.

[12] E. W. Kimbark, Power System Stability. New York: John Wiley \& Sons, 1947, vol. I.

[13] H. D. Chiang, C. C. Chu, and G. Cauley, "Direct stability analysis of electric power systems using energy functions: Theory, applications, and perspective," Proc. IEEE, vol. 83, no. 11, pp. 1497-1529, November 1995.
[14] IEEE/CIGRE Joint Task Force on Stability Terms and Definitions, "Definition and classification of power system stability," IEEE Trans. Power Syst., vol. 19, no. 2, pp. 1387-1401, May 2004.

[15] C. J. Tomlin, I. Mitchell, A. M. Bayen, and M. Oishi, "Computational techniques for the verification of hybrid systems," Proc. IEEE, vol. 91, no. 7, pp. 986-1001, July 2003.

[16] I. M. Mitchell, "Comparing forward and backward reachability as tools for safety analysis," in Hybrid Systems: Computation and Control, ser. Lecture Notes in Computer Science 4416, A. Bemporad, A. Bicchi, and G. Buttazzo, Eds. Springer-Verlag, 2007, pp. 428-443.

[17] S. N. Talukdar, J. Apt, M. Ilic, L. B. Lave, and M. G. Morgan, "Cascading failures: Survival versus prevention," The Electricity J., vol. 16, no. 9, pp. 25-31, November 2003 .

[18] G. Andersson, P. Donalek, R. Farmer, N. Hatziargyriou, I. Kamwa, P. Kundur, N. Martins, J. Paserba, P. Pourbeik, J. Sanchez-Gasca, R. Schulz, A. Stankovic, C. Taylor, and V. Vittal, "Causes of the 2003 major grid blackouts in North America and Europe, and recommended means to improve system dynamic performance," IEEE Trans. Power Syst., vol. 20, no. 4, pp. 1922-1928, November 2005.

[19] Y. Susuki and T. Hikihara, "Predicting voltage instability of power system via hybrid system reachability analysis," in Proc. American Control Conf., New York City, United States, July 11-13 2007, pp. 4166-4171.

[20] I. M. Mitchell and Y. Susuki, "Level set methods for computing reachable sets of hybrid systems with differential algebraic equation dynamics," in Hybrid Systems: Computation and Control, ser. LNCS 4981. Springer-Verlag, 2008, pp. 630-633.

[21] T. J. Koo and H. Su, "A computational approach for estimating stability regions," in Proc. IEEE Int. Symp. Computer-Aided Control Systems Design, Munich, Germany, October 2006.

[22] L. -J. Jin, H. -F. Liu, R. Kumar, J. D. McCalley, N. Elia, and V. Ajjarapu, "Power system transient stability design using reachability based stability-region computation," in Proc. North American Power Symp., 383-343, 2005.

[23] T. Hikihara, "Application of hybrid system theory to power system analysis (I)," in Annual Meeting Record, IEE Japan, vol. 6, March 2005, p. 187, (in Japanese).

[24] Y. Susuki, H. Ebina, and T. Hikihara, "Application of hybrid system theory to power system stability analysis," in Proc. Int. Symp. Nonlinear Theory and its Applications, Bruge, Belgium, October 2005, pp. 202205.

[25] - "Predicting transient instability of power systems based on hybrid system reachability analysis," in Preprints of the IFAC Symposium on Power Plants and Power Systems Control, Kananaskis, Canada, June 25-28 2006, article no. 599.

[26] Y. Susuki, T. Sakiyama, T. Ochi, T. Uemura, and T. Hikihara, "Verifying fault release control of power system via hybrid system reachability," in Proc. North American Power Symp., Calgary, Canada, September 28-30 2008.

[27] T. J. Koo, X. Qin, S. Yang, and Y. Susuki, "Reachability computation for power system transient stability," in Proc. Int. Symp. Nonlinear Theory and its Applications, Sapporo, Japan, October 18-21 2009, pp. 552-555.

[28] I. A. Hiskens and M. A. Pai, "Hybrid systems view of power system modeling," in Proc. IEEE Int. Symp. Circuits and Systems, vol. II, Geneva, Switzerland, May 2000, pp. 228-231.

[29] —_ "Trajectory sensitivity analysis of hybrid systems," IEEE Trans. Circuits Syst. I, vol. 47, no. 2, pp. 204-220, February 2000.

[30] I. A. Hiskens, "Power system modeling for inverse problems," IEEE Trans. Circuits Syst. I, vol. 51, no. 3, pp. 539-551, March 2004.

[31] T. Geyer, M. Larsson, and M. Morari, "Hybrid emergency voltage control in power systems," in Proc. European Control Conf., Cambridge, England, September 2003.

[32] A. G. Beccuti, T. Geyer, and M. Morari, "A hybrid system approach to power systems voltage control," in Proc. IEEE Conf. Decision and Control \& European Control Conf., Seville, Spain, December 2005, pp. 6774-6779.

[33] R. R. Negenborn, A. G. Beccuti, T. Demiray, G. Damm, B. D. Schutter, and M. Morari, "Supervisory hybrid model predictive control for voltage stability of power networks," in Proc. American Control Conf., New York City, USA, July 2007, pp. 5444-5449.

[34] S. Leirens, J. Buisson, P. Bastard, and J. L. Coullon, "A hybrid approach for voltage stability of power systems," in Proc. Power Systems Computation Conf., Liege, elgium, August 2005, article No. 16-1.

[35] S. A. Attia, M. Alamir, and C. Canudas de Wit, "Voltage collapse avoidance in power systems: A receding horizon approach," Intell. Autom. Soft Co., vol. 12, no. X, pp. 1-14, 2006. 
[36] G. K. Fourlas, K. J. Kyriakopoulos, and C. D. Vournas, "Hybrid systems modeling for power systems," IEEE Circuits Syst. Mag., vol. 4, no. 3, pp. 16-23, Third Quarter 2004.

[37] H. G. Kwatny, E. Mensah, D. Niebur, and C. Teolis, "Optimal shipboard power system management via mixed integer dynamic programming," in Proc. IEEE Electric Ship Technologies Symp., Philadelphia, USA, July 2005, pp. 55-62.

[38] _ - "Optimal power system management via mixed integer dynamic programming," in Preprints of the IFAC Symposium on Power Plants and Power Systems Control, Kananaskis, Canada, June 2006, article No. 556.

[39] Y. Susuki, Y. Takatsuji, and T. Hikihara, "Hybrid model for cascading outage in a power system: A numerical study," IEICE Trans. Fund. Electr., vol. E92-A, no. 3, pp. 871-879, March 2009.

[40] Y. Takatsuji and T. Hikihara, "A hybrid modeling and dynamics of virtual microgrid under balancing restriction," in Proc. Int. Symposium on Nonlinear Theory and its Applications, Sapporo, Japan, October 1821 2009, pp. 548-551.

[41] Y. Takatsuji, Y. Susuki, and T. Hikihara, "Hybrid controller for safe microgrid operation," Nonlinear Theory and its Applications, IEICE, vol. 2, no. 3, pp. 347-362, July 2011.

[42] A. Giani, S. Sastry, K. H. Johansson, and H. Sandberg, "The VIKING project: An initiative on resilient control of power networks," in Proc. Int. Symp. Resilient Control Systems, 2009.

[43] P. M. Esfahani, M. Vrakopoulous, K. Margellos, J. Lygeros, and G. Andersson, "Cyber attach in a two-area power system: Impact identification using reachability," in Proc. American Control Conf., Baltimore, USA, June 2010, pp. 962-967.

[44] T. A. Henzinger, "The theory of hybrid automata," in Proc. IEEE Symp. Logic in Computer Science, 1996, pp. 278-292.

[45] J. Lygeros, K. H. Johansson, S. N. Simić, J. Zhang, and S. S. Sastry, "Dynamical properties of hybrid automata," IEEE Trans. Automat. Contr., vol. 48, no. 1, pp. 2-17, January 2003.

[46] C. J. Tomlin, J. Lygeros, and S. S. Sastry, "A game theoretic approach to controller design for hybrid systems," Proc. IEEE, vol. 88, no. 7, pp. 949-970, July 2000.

[47] J. A. Sethian, Level Set Methods and Fast Marching Methods: Evolving Interfaces in Computational Geometry. Fluid Mechanics, Computer Vision, and Materials Science. Cambridge University Press, 1999.

[48] S. Osher and R. Fedkiw, Level Set Methods and Dynamic Implicit Surfaces. Springer, 2003.

[49] I. Mitchell and C. Tomlin, "Level set methods for computation in hybrid systems," in Hybrid Systems: Computation and Control, ser. Lecture Notes in Computer Science 1790, B. Krogh and N. Lynch, Eds. Springer-Verlag, 2000, pp. 310-323.

[50] A. Dubey, X. Wu, H. Su, and T. J. Koo, "Computation platform for automatic analysis of embedded software systems using model based approach," in Automated Technology for Verification and Analysis, ser. Lecture Notes in Computer Science 3707. Springer Berlin / Heidelberg, 2005, pp. 114-128.

[51] T. J. Koo, G. J. Pappas, and S. Sastry, "Mode switching synthesis for reachability specifications," in Hybrid Systems, ser. Lecture Notes in Computer Science 2034, M. D. D. Benedetto and A. SangiovanniVincentelli, Eds. Springer Berlin / Heidelberg, 2001, pp. 333-346.

[52] M. Minorsky, Introduction to Non-Linear Mechanics. Ann Arbor, USA: Edwards Brothers, 1947.

[53] I. Mitchell and C. J. Tomlin, "Overapproximating reachable sets by Hamilton-Jacobi projections," J. Sci. Comput., vol. 19, no. 1-3, pp. $323-$ 346, 2003.

PLACE

PHOTO

HERE
Yoshihiko Susuki received the Ph.D. degree in engineering from Kyoto University in 2005. In 2005 he joined to the Department of Electrical Engineering at Kyoto University, where he is currently Assistant Professor. In 2008-2010 he stayed in the Department of Mechanical Engineering at the University of California, Santa Barbara, where he was Visiting Researcher. His research interests are in power engineering, nonlinear dynamical systems, and control systems.

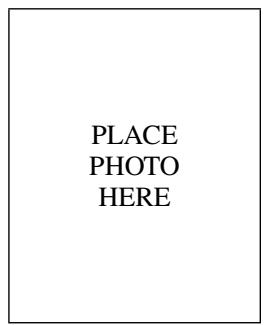

T. John Koo received the Ph.D. degree in electrical engineering from the University of California at Berkeley in 2000, and the M.Phil. degree in information engineering and the B.Eng. degree in electronic engineering from the Chinese University of Hong Kong in 1994 and 1992, respectively. He is Professor and Director of the Center for Embedded Software Systems, Shenzhen Institutes of Advanced Technology, Chinese Academy of Sciences. His research interests include embedded software, hybrid systems, nonlinear control, and robotics. Dr. Koo is the recipient of the National Science Foundation Faculty Early CAREER Development Award, Vanderbilt University, in 2005, and the Distinguished M.Phil. Thesis Award of the Faculty of Engineering, the Chinese University of Hong Kong, in 1994.

\begin{tabular}{|c|}
\hline \\
PLACE \\
PHOTO \\
HERE \\
\end{tabular}

Hiroaki Ebina received the master degree in electrical engineering from Kyoto University in 2007. He is currently with Kansai Electric Power Co., Inc., Japan. During his master course in Kyoto University, he worked on applications of hybrid systems theory to power system analysis and control.

Takuya Yamazaki (Sakiyama) received the master degree in electrical engineering from Waseda University in 2002 . He is currently with Kansai Electric Power Co., Inc., Japan.

Takashi Ochi graduated Miyakojima Technical Senior High School in 1982. $\mathrm{He}$ is currently with Kansai Electric Power Co., Inc., Japan.

Takuji Uemura received his master degree in engineering science from Osaka University in 1985 . He is currently with Kansai Electric Power Co., Inc., Japan.

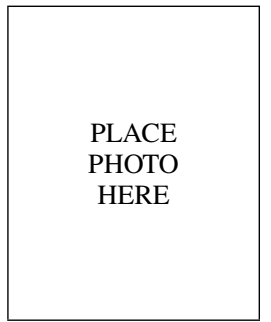

Takashi Hikihara received the Ph.D. degree in engineering from Kyoto University in 1990. In 19871997, he joined to Kansai University, where he was Research Associate, Assistant Professor, and Associate Professor. In 1993-1994, he stayed in the Sibley School of Mechanical and Aerospace Engineering at Cornel University, where he was Visiting Researcher. In 1997, he joined to the Department of Electrical Engineering at Kyoto University, where he is currently Professor and DDG of Kyoto University Library Network. His research interests are in power electronics, nonlinear dynamics, micro and nano-science, and control engineering. 